\title{
Somatosensory cortical remodeling after rehabilitation and clinical benefit of in writer's cramp
}

${ }^{1,3}$ Bleton* Jean Pierre, ${ }^{2,5,6,10}$ Vidailhet*Marie, ${ }^{4}$ Bourdain* Frédéric, ${ }^{5,6,7,10}$ Ducorps Antoine, ${ }_{5,6,7,10}$ Schwartz Denis, ${ }^{6,10,11}$ Delmaire Christine, ${ }^{5,6,10,12}$ Lehéricy Stéphane, ${ }^{5,6,7}$ Renault Bernard, ${ }^{5,6,7,10}$ Garnero Line ${ }^{\dagger},{ }^{5,8,9}$ Meunier Sabine

Authors contributed equally*

${ }^{1}$ Université Paris Descartes EA 4055/ISERM U894.

${ }^{2}$ AP-HP, Groupe hospitalier Pitié-Salpétrière, Fédération de Neurologie, Paris, F-75013

${ }^{3}$ Service de Neurologie, Hôpital Sainte-Anne, F-75005, Paris, France

${ }^{4}$ Service de Neurologie, Hôpital Foch, Suresnes, 92150 France

${ }^{5}$ UPMC University Pierre et Marie Curie-Paris6, Paris, F-75013

${ }^{6}$ CRICM, INSERM UMR_S 975, CNRS UMR 7225, Paris, F-75013

${ }^{7}$ Centre de Magnétoencéphalographie CNRS LENA UPR 640 Université Paris VI, Paris, F-5013

${ }^{8}$ UPMC Université Pierre et Marie Curie-Paris6, ER6, Physiologie et physiopathologie de la motricité chez l'homme Paris, F-75013

${ }^{9}$ Médecine Physique et Réadaptation, Hôpital Pitié-Salpêtrière, Paris

${ }^{10}$ Federative Institute of Research on Functional Neuroimaging (IFR49), NeuroSpin, 91191

Gif-sur-Yvette, France

${ }^{11}$ Service de Neuroradiologie, CRHU Lille, 59000 Lille, France

${ }^{12}$ CENIR - Centre de Neuroimagerie de Recherche, Groupe hospitalier Pitié-Salpétrière, Paris, F-75013, France

These authors contributed equally*

Key words: dystonia - writer's cramp - magnetoencephalography - rehabilitation somatotopy - plasticity - cortical mapping

Running title: Reshaping the cortical map in dystonia Words count: Abstract: 138; Text. 1499; 1 Table, 1 Figure; 21 references.

Correspondence: Pr Marie Vidailhet, Fédération de Neurologie, Hôpital de la Salpêtrière, 75013 Paris France Tel: + 33142162748 Fax: + 33142162478 E-Mail: marie.vidailhet@psl.aphp.fr 


\begin{abstract}
In order to explore the pathophysiological basis of a new rehabilitation therapy in writer's cramp (WC), healthy controls, untreated WC and WC who recovered a legible handwriting after rehabilitation were explored using magnetoencephalography and the somatosensory evoked fields of fingers I, II, III and V in the sensory cortex were studied. In the cortex controlling the dystonic limb, the size of the hand representation in the trained patients was similar to that of healthy controls, and significantly different from that of untrained patients. Trained patients exhibited "super-normal" reorganization of the finger maps. In the cortex controlling the non-dystonic limb, there was little difference between trained and untrained patients and the hand representation was enlarged and disorganized. We hypothesize that prolonged tailored rehabilitation in WC may induce long term plasticity phenomena, lateralized to the cortex controlling the dystonic hand.
\end{abstract}


Writer's cramp (WC) is a task-specific disorder associated with abnormal postures of the upper limb during writing. Injections of botulinum toxin can be partially effective in a sub-group of patients but may be disappointing on the long term. ${ }^{1}$ A more recent approach is based on neurorehabilitation to target underlying pathophysiological processes such as degraded cortical and subcortical representations, ${ }^{2}$ impaired sensori-motor integration and maladaptative plasticity. ${ }^{3,4}$

In keeping with the view that abnormal plasticity may underlie the dystonic symptoms, we developed a rehabilitation program using targeted repetitive peripheral sensory stimulations during the execution of skilled manual tasks, a crucial process to promote the development of LTP/LTD in the M1 cortex. ${ }^{5,6}$ Moreover, as an aberrant sensory-cued motor program with inappropriately scaled and gated input from the S1 cortex may lead to inadequately patterned activation/deactivation of the M1 cortex ${ }^{7}$, we also used a stepwise, fragmented and individually tailored motor training. We hypothesize that behavioral treatments of dystonia may modify the functional organization of central somatosensory networks. Our study was aimed to explore plasticity phenomena of the cortical map associated with rehabilitation in WC. Therefore, we specifically selected WC who had recovered near-normal handwriting after rehabilitation. We studied them once their writing performance had stabilized to ensure that the physiological correlates we examined were not related to short term plasticity rapidly induced by manipulation of somatosensory inputs ${ }^{8}$ but to a relearning process. Finger representation in the S1 cortex, mapped by using magnetoencephalography (MEG), was compared with those of untreated WC (who had undergone neither rehabilitation nor botulinum toxin injections).

\section{METHODS}

Eleven patients with previously disabling WC (group R: 8 women, 3 men; age $43.3 \pm 14$ years, (22-64); mean $\pm \mathrm{SD}$, range, (years), who recovered fluent writing with close to normal posture after rehabilitation (subjective improvement and writing score on the Burke-Fahn-Marsden disability scale (BFM), ${ }^{9}$ were compared with 10 untreated WC (group D: 6 women, 4 men; age $48.7 \pm 8$ (28-60 years), and with 11 healthy controls (group C: 7 women, 4 men; age $43 \pm 13$ (2771 years). Groups D and R were matched for the duration of illness (R: $5.6 \pm 5.4$ years (1-13); D: mean $4.1 \pm 3.2$ years (1-10), Mann-Whitney $\mathrm{U}$ test $p=0.7$ ) movement score on BFM (R before rehabilitation, mean $2.2 \pm 0.7$ (1-3); D, mean $1.9 \pm 0.7$ (1-3), Mann-Whitney U test $p=0.4$ ). All patients were evaluated by the same neurologists (MV, FB) at the time of the study and before and after rehabilitation. The rehabilitation program was a re-learning process with weekly 30 minutes individual sessions with a physiotherapist (JPB) and daily similar home program, for 
several months (average 5.5 months). It included relaxation techniques, correction of elementary movement not directly pertaining to writing activity, training of finger dexterity and ergonomic postures, sensory control (pressure, position on the pen), synergies between proximal and distal muscles, progression from simple to complex graphic exercises drawing and writing) and fractionated epochs of work with rest and relaxation.

Right and left hand representations were acquired by MEG and 3D-MRI 3D brain reconstructions as previously described ${ }^{2}$ and data analysis was done using the same quantitative measures, Dm and Dmax. A somatotopy index (SI) allowed us to assess semi quantitatively the topographic order of the fingers. The study was approved by the Ethical Committee of the Salpêtrière Hospital, Paris, France.

\section{Statistical analysis}

The effects of the group $(\mathrm{C} / \mathrm{D} / \mathrm{R})$, the side (dominant/non dominant hand) and their interaction were assessed by using 3-group analysis of variance (ANOVA) with Dmax, Dm and SI as dependant variables. Post-hoc comparisons were done using Fisher's test. If there was a significant effect of side, the effect of the group was tested separately for each hand by ANOVA. The effect of rehabilitation on the clinical scores was tested by repeated ANOVA with the two values of the clinical scores (before and after rehabilitation) forming the repeats.

\section{RESULTS}

Clinical assessment:

With rehabilitation, there was an improvement in the BFM movement (from $2.36 \pm 0.7$ to $0.64 \pm$ 0.7; repeated measure ANOVA, $p<0.0001$ ) and disability (from $1.82 \pm 0.7$ to $0.18 \pm 0.4, p<$ 0.0001 ) scores. Patients in group $\mathrm{R}$ were trained for a mean of 5.5 months (range 2-12 months). Improvement of handwriting is illustrated in the Table. The degree of improvement was not influenced by the time ( 1 month to 1 year) elapsed since the end of rehabilitation $(r 2=0.007)$.

\section{Quantitative assessment of hand representation in S1 (Table)}

Dm was similar in rehabilitated patients $(\mathrm{R})$ and controls $(\mathrm{C})$, and was significantly larger in the untreated WC (D) (ANOVA: effect of group $p<0.0012$, effect of side $p<0.015$, group*side interaction $p=0.5$ (post-hoc Fisher tests: $\mathrm{D}$ versus $\mathrm{R} p<0.0004$, $\mathrm{R}$ versus $\mathrm{D} p<0.006$, $\mathrm{R}$ versus $\mathrm{C} p n s$ ). This was found in both hemispheres (Fisher test, dominant hemisphere $\mathrm{C}$ versus $\mathrm{R} p=$ $0.6, \mathrm{C}$ versus $\mathrm{D} p<0.02$, D versus $\mathrm{R} p<0.05$; non-dominant hemisphere $\mathrm{C}$ versus $\mathrm{R} p=0.4, \mathrm{C}$ versus $\mathrm{D} p<0.01$, D versus $\mathrm{R} p<0.04)$. 
There was a trend towards significance for Dmax among the 3 groups (ANOVA effect of group $p=0.06$ ). Dmax was significantly different between the right and left side (effect of side $p<0.01$, interaction side*group $p n s$ ). We thus tested each hemisphere separately. In the non-dominant hemisphere, non rehabilitated patients had a larger Dmax than healthy volunteers and rehabilitated patients ( $\mathrm{D}$ versus $\mathrm{C} p<0.008, \mathrm{D}$ versus $\mathrm{R} p<0.05$, $\mathrm{R}$ versus $\mathrm{C} p n s$ ).

\section{Topographic order of the fingers (Figure, Table)}

SI differed between groups C, D and R, with a side*group interaction (ANOVA group $p<0.05$, group*side $p<0.02$ ). This interaction was due to a higher SI index in the dominant hemisphere of the rehabilitated patients versus control and WC (Fisher test: D versus $\mathrm{R} p<0.0005, \mathrm{C}$ versus $\mathrm{R} p$ $<0.003, \mathrm{C}$ versus $\mathrm{D} p=0.5)$. The SI index in the left post-central gyrus of the group $\mathrm{R}$ patients was close to $3(\mathrm{SI}=2.7)$ as the order of the fingers fitted the Penfield homuncular organization. In controls, the left post-central gyrus order of finger representations was more variable (SI = 1.7), with frequent inversions of two adjacent finger representations, as previously described. ${ }^{2}$. SI did not differ between the three groups in the non-dominant hemisphere.

\section{Discussion}

In specifically selected WC who recovered near-normal handwriting after prolonged rehabilitation and had stabilized their writing performance, the MEG somatosensory map of the cortex controlling the dystonic hand is "normalized" as the size of hand representation of these rehabilitated WC was similar to that of healthy controls and significantly different from that of untreated WC. Moreover, the order of the fingers fitted the Penfield homuncular organization, creating a "super normal" somatotopic order of the fingers (in normal subjects, the order of finger representations could be slightly variable with inversions of two adjacent finger representations). ${ }^{2}$ In line with these results, we hypothesize that prolonged tailored rehabilitation in WC may induce long term plasticity phenomena, lateralized to the cortex controlling the dystonic hand. Moreover, it may persist over time as the patients were studied long after the end of the retraining process. In addition, the endophenotype of dystonia ${ }^{2}$ would still be detectable in the cortex controlling the non dystonic hand, with a disruption of the MEG somatosensory map of the hand, similar in rehabilitated and untreated patients.

Several studies, with various methods of clinical evaluation, reported some beneficial effects of somatosensory training, ${ }^{10,} 11$ sensori-motor retuning, ${ }^{12-14}$ and learning-based sensori-motor

training ${ }^{15-17}$ in $\mathrm{WC}^{18,} 19$ or musician's dystonia. ${ }^{12,18-20}$ Few studies have examined the physiological correlates of rehabilitation strategies WC. In most of them, the training program was 
brief and patients were tested shortly after. No change in motor or sensory cortical excitability were observed after 4 weeks of motor training. ${ }^{13}$ Changes in short-interval-intracorticalinhibitition were found after 15 minutes of proprioceptive training. ${ }^{21}$ The somatosensory map of the hand before and immediately after 8 consecutive days of sensorimotor retraining therapy in musicians was studied using MEG recordings in one study. ${ }^{12}$ After rehabilitation, finger representations contralateral to the dystonic side became similar to those of the non affected side, whereas they differed before treatment. As this effect was observed immediately after the end of a short training period this may mainly reflect short term plasticity. In contrast, we may have explored long term plasticity phenomena as we examined cortical maps up to 1 year after the end of a prolonged (5.5 months) rehabilitation program. The lack of MEG mapping prior to rehabilitation is a limitation in our study. Ideally, larger groups of patients would have allowed to compare patients who have been through similar rehabilitation program and had various amount of improvement and to study the correlations between clinical improvement and finger cortical representations. Our work may be considered as a pilot, preliminary study, contributing to the field of neuro-rehabilitation with a new rehabilitation program leading to sustained clinical, benefit potentially reflecting long-term plasticity.

\section{Acknowledgements}

This work was supported by the INSERM National Dystonia Network,GIS-Maladies Rares and the patients' association AMADYS.

Dr F Bourdain was supported by a grant from Société Française de Neurologie.

Dr Sabine Meunier was supported by grants from AP-HP, INSERM and MESR (Unité de recherche mixte U731 INSERM/UPMC), IRME and Institut Garches.

We are indebted to Drs Sangla and Trocello for patient recruitment and to Pr. Dormont for his help in MRI acquisitions.

We dedicate this work to Dr Line Garnero $\dagger$, $\mathrm{PhD}$, late Director of the MEG centre.

the Corresponding Author has the right to grant on behalf of all authors and does grant on behalf of all authors, an exclusive licence on a worldwide basis to the BMJ Publishing Group Ltd and its Licensees to permit this article (if accepted) to be published in the Journal of Neurology, Neurosurgery \& Psychiatry editions and any other BMJPGL products to exploit all subsidiary rights, as set out in our licence (http://jnnp.bmjjournals.com/ifora/licence.pdf). 


\section{Legends:}

Figure: Finger somatotopy in the primary sensory cortex and sample of handwriting prior and after rehabilitation

The equivalent current dipole locations corresponding to each finger (thumb-I (yellow dot), indexII (green dot) middle-III (blue dot) and little-V fingers (red dot)) are superimposed on 3D brain reconstructions in a control subject (A), a non-rehabilitated dystonic patient (B) and a rehabilitated dystonic patient (C). Sample of handwriting and drawing before and 2.5 months after rehabilitation (D). WC: writer's cramp

\section{Table: Quantitative assessment of hand area in primary sensory cortex}

R: rehabilitated patients with writer's cramp. D: untrained patients with writer's cramp. C: normal control subjects. Dm: mean Euclidean distance between the equivalent current dipole (ECD) locations of two adjacent fingers in the inferior-superior direction in the sagittal plane; Dmax: Euclidean distance between the ECD locations of the two fingers most distant from one another. SI: somatotopy index indicating the correct topographic order of adjacent fingers. The SI was 3 if the finger ECDs hada correct topographic order in the inferior-superior direction in the sagittal plane (I, II, III, V from bottom to top); The SI was 2 if there was an inversion between the ECDs of adjacent fingers; and the SI was 1 if there were 2 or 3 inversions, or an inversion between two nonadjacent fingers. Values are expressed as mean $+\mathrm{SD}$. 


\section{References}

1. Kruisdijk JJ, Koelman JH, Ongerboer de Visser BW et al. Botulinum toxin for writer's cramp: a randomised, placebo-controlled trial and 1-year follow-up. J Neurol Neurosurg Psychiatry. 2007;78:264-270

2. Meunier S, Garnero L, Ducorps A et al. Human brain mapping in dystonia reveals both endophenotypic traits and adaptive reorganization. Ann Neurol. 2001;50:521-527

3. Vidailhet M, Grabli D, Roze E. Pathophysiology of dystonia. Curr Opin Neurol.2009;22:406413

4. Breakefield XO, Blood AJ, Li Y et al. The pathophysiological basis of dystonias. Nat Rev Neurosci. 2008;9:222-234

5. Kaelin-Lang A, Luft AR, Sawaki L et al. Modulation of human corticomotor excitability by somatosensory input. J Physiol. 2002;540:623-633

6. Quartarone A, Siebner HR, Rothwell JC. Task-specific hand dystonia: can too much plasticity be bad for you? Trends Neurosci. 2006;29:192-199

7. Lemon RN. Functional properties of monkey motor cortex neurones receiving afferent input from the hand and fingers. J Physiol. 1981;311:497-519

8. Wang X, Merzenich MM, Sameshima K, Jenkins WM. Remodelling of hand representation in adult cortex determined by timing of tactile stimulation. Nature. 1995;378:71-75

9. Burke RE, Fahn S, Marsden CD et al. Validity and reliability of a rating scale for the primary torsion dystonias. Neurology. 1985;35:73-77

10. Zeuner KE, Bara-Jimenez W, Noguchi PS et al. Sensory training for patients with focal hand dystonia. Ann Neurol. 2002;51:593-598

11. Tinazzi M, Fiorio M, Fiaschi A et al. Sensory functions in dystonia: insights from behavioral studies. Mov Disord. 2009;24:1427-1436

12. Candia V, Wienbruch $\mathrm{C}$, Elbert $\mathrm{T}$ et al. Effective behavioral treatment of focal hand dystonia in musicians alters somatosensory cortical organization. Proc Natl Acad Sci USA. 2003;100:7942-7946

13. Zeuner KE, Shill HA, Sohn YH et al. Motor training as treatment in focal hand dystonia. Mov Disord. 2005;20:335-341

14. Zeuner KE, Molloy FM. Abnormal reorganization in focal hand dystonia--sensory and motor training programs to retrain cortical function. NeuroRehabilitation. 2008;23:43-53

15. Schenk T, Bauer B, Steidle B, Marquardt C. Does training improve writer's cramp? An evaluation of a behavioral treatment approach using kinematic analysis. J Hand Ther. 2004; 17:349-363

16. Baur B, Furholzer W, Jasper I et al. Effects of modified pen grip and handwriting training on writer's cramp. Arch Phys Med Rehabil. 2009;90:867-875

17. Byl NN, Archer ES, McKenzie A. Focal hand dystonia: effectiveness of a home program of fitness and learning-based sensorimotor and memory training. J Hand Ther. 2009;22:183-197

18. Byl NN, Nagajaran S, McKenzie AL. Effect of sensory discrimination training on structure and function in patients with focal hand dystonia: a case series. Arch Phys Med Rehabil. 2003;84:1505-1514

19. McKenzie AL, Goldman S, Barrango $\mathrm{C}$ et al. Differences in physical characteristics and response to rehabilitation for patients with hand dystonia: musicians' cramp compared to writers' cramp. J Hand Ther. 2009;22:172-181; quiz 182

20. Candia V, Rosset-Llobet J, Elbert T, Pascual-Leone A. Changing the brain through therapy for musicians' hand dystonia. Ann N Y Acad Sci. 2005;1060:335-342

21. Rosenkranz K, Butler K, Williamon A et al. Sensorimotor reorganization by proprioceptive training in musician's dystonia and writer's cramp. Neurology 2008;70:304-315 
Figure. Finger somatotopy in the primary sensory cortex and sample of handwriting

A

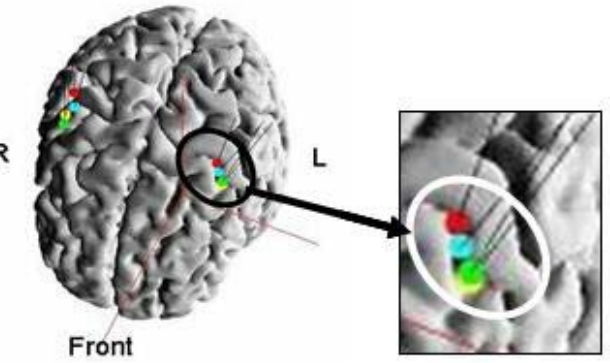

Healthy subject normal somatotopic order of finger representation

B

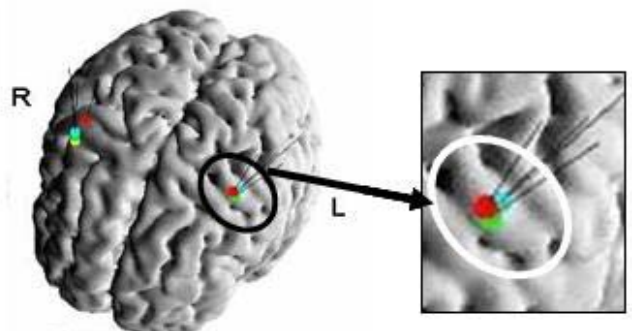

Untreated WC disorganised somatotopic order of finger representation

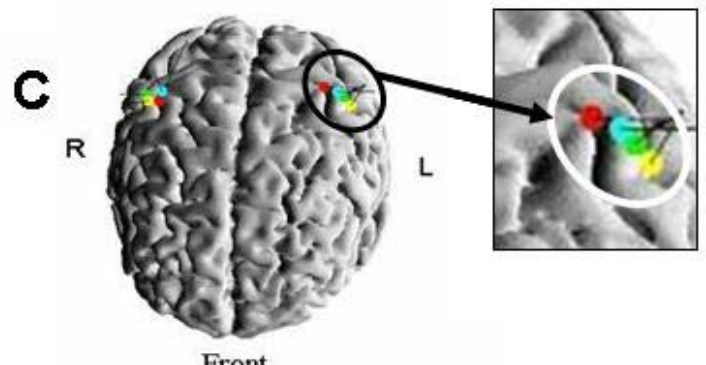

\section{Rehabilitated WC "super-normal" somatotopic order of finger representation}

D

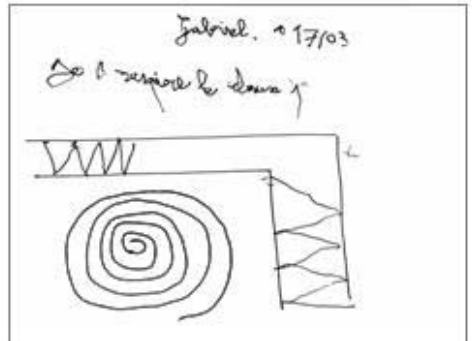

before rehabilitation

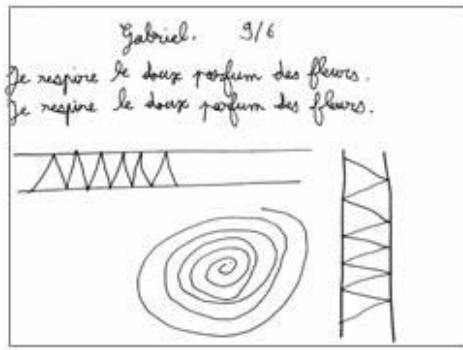

after 2.5 months of rehabilitation 
Table - Quantitative assessment of hand area in primary sensory cortex

Table - Quantitative assessment of hand area in primary sensory cortex

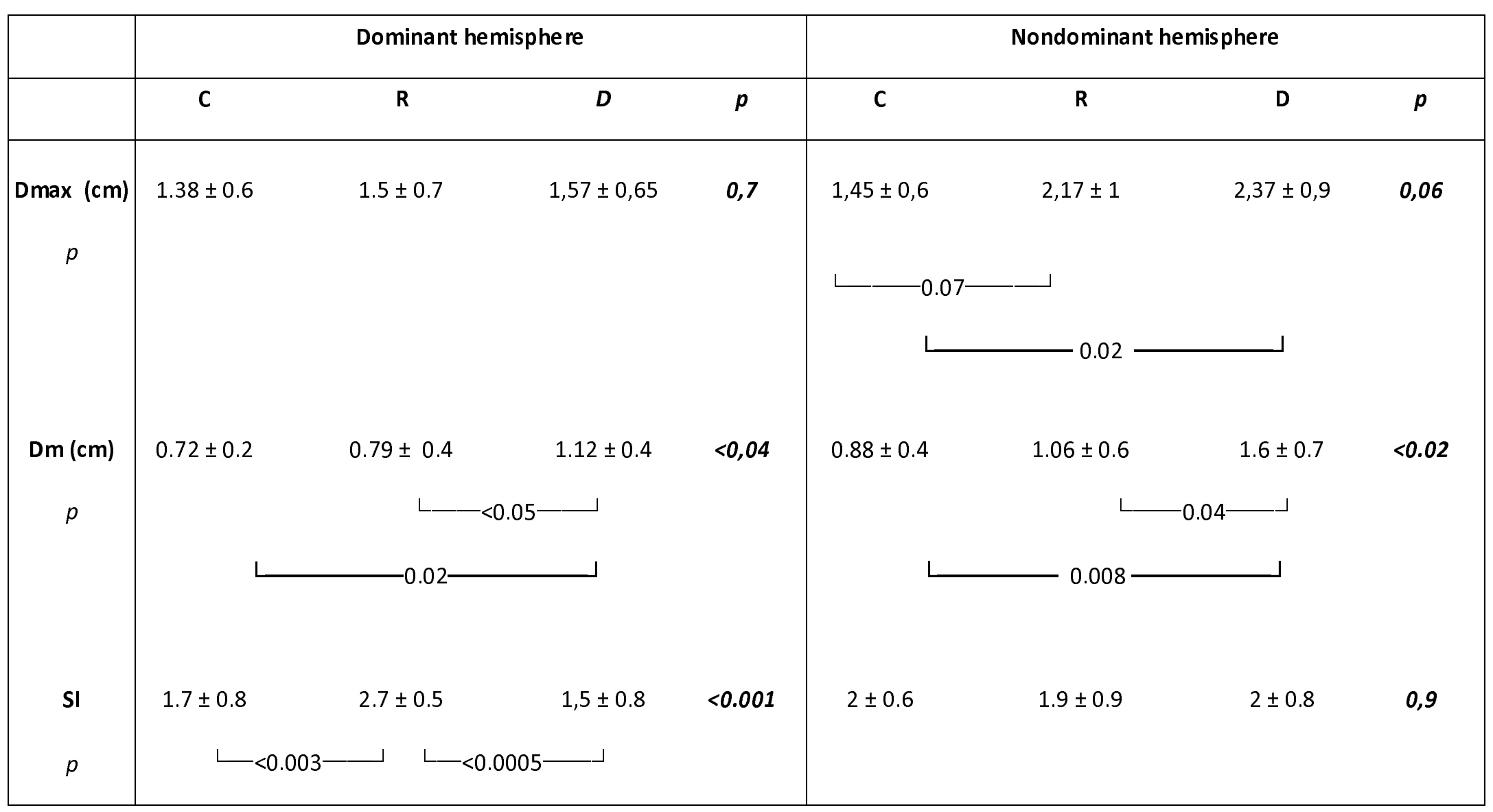


A

n

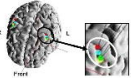

Hoalthy sLbjest

กजัต।

vernatologic ordor of

firger icpresentetian

$B$

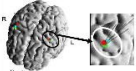

\section{Untreatod WC ciscraarised} scrnataboic order of inger requesertiotion

Irang

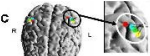

Rahabilitatod WC

'supornomal'

comototepis ander of

finger raprosematián

D

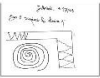

beíre retabitation

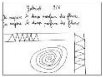

atter 2.5 merthe of rahabiltation 